\title{
Effect of dialysis duration on serum calcium and phosphorous in hemodialysis patients
}

\begin{abstract}
In many countries, physicians follow clinical guidelines for mineral bone disorders to control secondary hyperparathyroidism associated with serum calcium and phosphorous level in patients maintaining hemodialysis (HD). ${ }^{1}$ The current research was conducted among 34 patients with chronic renal insufficiency on maintenance HD to study serum Phosphorous, Calcium, Alkaline phosphatase changes in maintenance hemodialysed patients.
\end{abstract}

Keywords: renal insufficiency, renal dialysis, calcium, phosphorous, biomarkers

\author{
Volume 2 Issue 3 - 2018 \\ Seyyed Mohsen Hosseininejad, 1,6 Mehrdad \\ Aghaei, ${ }^{2,6}$ Gholamreza Roshandel, ${ }^{3}$ Fateme \\ Sadat Hosseininejad, ${ }^{4}$ Atiyeh Moazzeni, ${ }^{5}$ \\ Robabeh Salehi, ${ }^{6,7}$ Saeid Amirkhanlou ${ }^{5,6,7}$ \\ 'Student Research Committee, Golestan University of Medical \\ Sciences, Gorgan, Iran \\ ${ }^{2}$ Golestan Rheumatology Research Center, Golestan University \\ of Medical Sciences, Gorgan, Iran \\ ${ }^{3}$ Golestan Research Center of Gastroenterology and \\ Hepatology, Golestan University of Medical Sciences, Gorgan, \\ Iran \\ ${ }^{4}$ Student Research Committee, School of Public Health, \\ Shahroud University of Medical Sciences, Shahroud, Iran \\ ${ }^{5}$ Hemodialysis Ward, 5th Azar Hospital, Golestan University of \\ Medical Sciences, Gorgan, Iran \\ ${ }^{6}$ Clinical Research Development Unit (CRDU), Sayyad Shirazi \\ Hospital, Golestan University of Medical Sciences, Gorgan, Iran \\ ${ }^{7}$ Hemodialysis Ward, Sayyad Shirazi Hospital, Golestan \\ University of Medical Sciences, Gorgan, Iran
}

Correspondence: Saeid Amirkhanlou, Clinical Research Development Unit (CRDU), Sayyad Shirazi Hospital, Golestan University of Medical Sciences, Gorgan, Iran, Tel 00989398387874, Email Drsam74ir@ymail.com

Received: June 06, 2018 | Published: June 18, 2018
Abbreviations: P, Phosphorous; Ca, Calcium; ALP, alkaline phosphatase; BMD, bone mineral density; CKD-MBD, chronic kidney disease-mineral and bone disorder; HD, hemodialysis; ESRD, end stage renal disease

\section{Introduction}

Chronic renal insufficiency is associated with changes in mineral and bone metabolism biochemistry and density. ${ }^{2,3}$ In many countries, physicians follow clinical guidelines for mineral bone disorders to control secondary hyperparathyroidism associated with serum calcium and phosphorous level in patients maintaining hemodialysis (HD). ${ }^{1}$ With the begin of hemodialysis, histological marks of secondary hyperparathyroidism could be seen in bones of over 50 percent $^{4}$ of end stage renal disease (ESRD) patients, skeletal defects identified as Chronic Kidney Disease-Mineral and Bone Disorder (CKD-MBD) (the so called as renal osteodystrophy) consist of some types of bone tissue lesions, such as the most widespread high bone turn-over disease but also the a dynamic bone disease.$^{5,6}$

Other less common bone diseases such as osteomalacia, aluminumrelated bone disease, fluorosis and strontium overload or mixed types also have been pronounced. ${ }^{4}$

It is well recognized that bone mineral density (BMD) is decreased in patients suffering chronic renal failure and they have higher fracture risk. ${ }^{7,8}$ Uremic patients frequently reveal high serum concentration of biochemical markers. ${ }^{3}$
The aim of current research is to study serum Phosphorous (P), Calcium (Ca), alkaline phosphatase (ALP) changes and other indices in hemodialysed patients.

\section{Methods \\ Participants}

Thirty four patients, 16 postmenopausal female and 18 male, on maintenance HD were involved in the current study after obtaining their informed consent. The study was conducted in Golestan University of Medical Sciences, Gorgan, Iran during the period February-May 2018. Patients mean age was 57.8 with range of $43-73$ years old, with the mean HD duration of 40 months (range: 11-204 months). All of our patients were treated by conventional HD 3-4 hours, three times in a week. None of the patients had a past history of renal transplantation or parathyroidectomy, history of radiographic evidence of rib, vertebral or hip fracture. At the time of the assessment, none of the patients, especially the postmenopausal women, were receiving or had received previously oestrogen or raloxifene, bisphosphonates, calcitonin, PTH or corticosteroids agents. None of the participants took vitamin $\mathrm{K}$ or aluminum hydroxide. There was no clinical nor biological sign for other bone disease such as osteomalacia, Paget's disease, malignancy or liver diseases.

\section{Biochemistry}

Morning pre-midweek hemodialysis blood samplings were 
collected from the arteriovenus fistula after a $12 \mathrm{hr}$ fasting. The serum obtained after centrifugation was kept in aliquots at $-20^{\circ} \mathrm{C}$ until analyzed, with measurements made instantly after defrosting.

Serum calcium, phosphorus, creatinine, and total alkaline phosphatase etc. were assessed routinely using an automatic analyzer.

\section{Statistics}

All obtained results are presented as mean $\pm \mathrm{SD}$, unless otherwise indicated. Correlations between variables were calculated using simple linear regression and $\mathrm{p}<0.05$ was considered as statistically significant. Comparison of variables was done using chi-square and/ or student t-test analysis.

\section{Results}

Demographic and biochemical data of thirty four patients, consisting of 18 male and 16 postmenopausal female, on maintenance HD were investigated.

Table 1 depicts the demographic data for the 34 patients and table 2 for the 18 male patients. Table 2 represents the biochemical data for all 34 patients.

The results of our study also showed that with dialysis increase, the serum biomarkers of $\mathrm{Ca}, \mathrm{P}$ and Alkaline phosphatase decrease; the correlations were statistically significant.

Table I Age and time-on-dialysis of the participants

\begin{tabular}{lll}
\hline & Mean \pm SD & Range (Yrs.) \\
\hline Age(year-old) & $57.8 \pm 12$ & $3 \mathrm{I}-78$ \\
Duration of dialysis(month) & $40.2 \pm 48$ & $\mathrm{II}-204$
\end{tabular}

Table 2 Biochemical data in all patients

\begin{tabular}{lll}
\hline & Mean & Std. Deviation \\
\hline Dialysis duration(month) & 40.29 & 48.3 \\
Calcium (mg/dl) & 8.53 & 0.79 \\
Phosphorus (mg/dl) & 5.812 & 1.72 \\
Alkaline phosphatase (U/l) & 462.18 & 202.76 \\
Hemoglobin(mg/dl) & 1.14 & 1.46 \\
Creatinine $(\mathrm{mg} / \mathrm{ml})$ & 8.65 & 2.13 \\
Sodium (mEq/l) & 139.18 & 3.34 \\
Fasting Blood Sugar(mg/dl) & 106 & 47.87 \\
Platelet $(* 103 \mu \mathrm{l})$ & 173.18 & 68.13 \\
\hline
\end{tabular}

\section{Discussion}

The existed data about the influence of hemodialysis among ESRD patients is limited; most of the studies have been dedicated to predialysis, HD and renal transplanted patients and occasional information is accessible for patients on peritoneal dialysis, hemofiltration and hemodialfiltration. ${ }^{9-11}$

In our study simple regression analysis showed no correlation between serum sodium and dialysis duration. Similarly, no correlation was found between age and serum biomarkers.

Serum $\mathrm{Ca}$ and $\mathrm{P}$ displayed a significant correlation only with duration of $\mathrm{HD}$ as expected which were in line with other previous reports. ${ }^{1,11-13}$ The differences between our results in comparison with other previous studies may be because of our limited sample size.

\section{Conclusion}

Remarkably, the duration of hemodialysis does act to disturb bone related serum biomarkers like ALP, $\mathrm{Ca}$, and $\mathrm{P}$; with some patients remaining outside the standard range for serum calcium and phosphorus range, practical guidelines and adequate treatment should be followed to reduce any other further risk of secondary hyperparathyroidism and its complications. Besides, the impact of other factors on bone metabolism and bone mineral density changes in HD patients importantly remains to be illuminated with further studies.

\section{Acknowledgments}

We'd like to appreciate the all Hemodialysis Ward Staff of Sayyad Shirazi Hospital and 5thAzar Hospitals (Gorgan, Iran) for their sincere contribution to the study.

\section{Conflict of interest}

The author declares there is no conflict of interest.

\section{References}

1. Kim G, Choi B, Cha DR, et al. Serum calcium and phosphorous level in patients undergoing maintenance hemodialysis: a multicenter study in Korea. Kidney Res Clin Pract. 2014;33(1):52-57.

2. Hruska KA, Teitelbaum SL. Mechanisms of disease: renalosteodystrophy N Engl J Med. 1995;333(3):166-174.

3. Yamamoto N. Morphological analysis of bone dynamics and metabolic bone disease. Bone Histomorphometry in CKD-MBD (chronic kidney disease mineral bone disorder. Clin Calcium. 2011;21(4):589-592.

4. De Vernejoul MC, Kuntz D, Miravet L, et al. Bone histomorphometry in hemodialysed patients. Metab Bone Dis Rel Res. 1981;3(3):175-179.

5. Yamamoto N. Morphological analysis of bone dynamics and metabolic bone disease. Bone Histomorphometry: the basic methods and role of bone research and clinical significance. Clin Calcium. 2011;21(4):529 533

6. Sherrard D, Hercz G, Pei Y. The spectrum of bone disease in end stage renal failure-an evolving disorder. Kidney Int. 1993;43(2):436-442.

7. Taal M, Masud T, Green D, et al. Risk factors for reduced bone density in haemodialysis patients. Nephrol Dial Transplant. 1999;14(8):19221928 .

8. Atsumi K, Kushida K, Yamazaki K, et al. Risk factors for vertebral fractures in renal osteodystrophy. Am J Kidney Dis. 1999;33(2):287293.

9. Zayour D, Daouk M, Medawar W, et al. Predictors of bone mineral density in patients on hemodialysis. Transplant Proc. 2004;36(5):12971301.

10. Urena P, Bernard-Poenaru O, Ostertag A. Bone mineral density, biochemical markers and skeletal fractures in haemodialysis patients. Nephrol Dial Transplant. 2003;18(11):2325-2331.

11. Doumouchtsis KK, Kostakis AI, Doumouchtsis SK, et al. Associations between osteoprotegerin and femoral neck BMD in haemodialysis patients. J Bone Miner Metab. 2008;26(1):66-72.

12. Negri A, Álvarez-Quiroga M, Bravo M. Estimation of the prevalence of low turnover renal osteodystrophy using biochemical markers in a peritoneal dialysis population. Nefrologia. 2001;21(4):392-394.

13. Polymeris A, Doumouchtsis K, Grapsa E. Bone mineral density and bone metabolism in hemodialysis patients. Correlation with PTH 25OHD3 and leptin. Nefrologia. 2012;32(1):73-78. 